\title{
Shannon Wavelet Analysis
}

\author{
Carlo Cattani \\ DiFarma, Università di Salerno \\ Via Ponte Don Melillo \\ 84084 Fisciano (SA)-Italy \\ ccattani@unisa.it
}

\begin{abstract}
In this paper the differentiable structure of the Shannon wavelets is defined and the projection of a linear differential operators is given for any order. As application, the wavelet solution of a heat propagation problem is computed and the contribution of the different scale components is explicitly shown.
\end{abstract}

Keywords: Shannon Wavelet, Connection coefficients, Heat equation, Numerical approximation.

AMS-Classification - 42C40, 42C15, 65T60, 35K05.

\section{Introduction}

Shannon wavelets are the real part of the so-called harmonic wavelets 245]. They have a slow decay in the variable space but a very sharp compact support in the frequency (Fourier) domain, being represented therein by box functions. This fact, together with the Parseval equality have been used to easily compute the inner product and the connection coefficients of the Shannon wavelets (see [23]) which are the inner product of the Shannon basis with their $\ell$-order derivatives. These coefficients, also called refinable integrals, are a very useful tool for the analysis of discrete samples [7/8] and for the derivation of the wavelet solution of partial differential equation (in the Petrov-Galerkin approach) [16] In the following the representation of linear operators in Shannon wavelet bases will be given. As an example the solution of the heat equation for a localized initial profile [6] will be obtain as a Shannon wavelet series. The main advantages of this approach are that: 1) only by using localized functions, like Shannon wavelets, the initial profile (and then its evolution in time), can be performed by the lowest number of series coefficients; 2) only with this approach the evolution can be split into many scales: some for the low frequency evolution and some for the high frequency evolution 3) the numerical approach is the simplest one.

\footnotetext{
* Work partially supported by Regione Campania under contract "Modelli nonlineari di materiali compositi per applicazioni di nanotecnologia chimica-biomedica", LR 28/5/02 n. 5, Finanziamenti 2003 and by Miur under contract "Modelli non Lineari per Applicazioni Tecnologiche e Biomediche di Materiali non Convenzionali", Univ. di Salerno, Processo di internazionalizzazione del sistema universitario, D.M. 5 agosto 2004 n. 262 - ART. 23.
} 


\section{Shannon Wavelets}

The dilated and translated instances of the Shannon scaling functions $\varphi_{k}^{n}(x) \equiv$ $2^{n / 2} \varphi\left(2^{n} x-k\right)$ and Shannon wavelet functions $\psi_{k}^{n}(x) \equiv 2^{n / 2} \psi\left(2^{n} x-k\right)$ are [3]:

$$
\left\{\begin{array}{l}
\varphi_{k}^{n}(x)=2^{n / 2} \frac{\sin \pi\left(2^{n} x-k\right)}{\pi\left(2^{n} x-k\right)} \\
\psi_{k}^{n}(x)=2^{n / 2} \frac{\sin \pi\left(2^{n} x-k-\frac{1}{2}\right)-\sin 2 \pi\left(2^{n} x-k-\frac{1}{2}\right)}{\pi\left(2^{n} x-k-\frac{1}{2}\right)} .
\end{array}\right.
$$

Their Fourier transform are

$$
\left\{\begin{array}{l}
\widehat{\varphi}_{k}^{n}(\omega)=\frac{2^{-n / 2}}{2 \pi} e^{-i \omega k / 2^{n}} \chi\left(\omega / 2^{n}+3 \pi\right) \\
\widehat{\psi}_{k}^{n}(\omega)=-\frac{2^{-n / 2}}{2 \pi} e^{-i \omega(k+1 / 2) / 2^{n}}\left[\chi\left(\omega / 2^{n-1}\right)+\chi\left(-\omega / 2^{n-1}\right)\right]
\end{array}\right.
$$

where the characteristic function $\chi(\omega)$, is defined as

$$
\chi(\omega) \equiv\left\{\begin{array}{l}
1,2 \pi \leq \omega<4 \pi \\
0, \text { elsewhere }
\end{array}\right.
$$

The family of functions $\left\{\psi_{k}^{n}(x)\right\}$ is an orthonormal wavelet basis with respect to the inner product, defined as

$$
\langle f, g\rangle \equiv \int_{-\infty}^{\infty} f(x) \overline{g(x)} \mathrm{d} x
$$

which, according to the Parseval equality, can be expressed as

$$
\langle f, g\rangle \equiv \int_{-\infty}^{\infty} f(x) \overline{g(x)} \mathrm{d} x=2 \pi \int_{-\infty}^{\infty} \widehat{f}(\omega) \overline{\widehat{g}(\omega)} \mathrm{d} \omega=2 \pi\langle\widehat{f}, \widehat{g}\rangle,
$$

where the bar stands for the complex conjugate.

The derivatives of the basis are fundamental tools for the computation of the $n$-order moments of the Shannon wavelets and connection coefficients:

$$
\gamma_{k h}^{(\ell) n m} \equiv\left\langle\frac{\mathrm{d}^{\ell}}{\mathrm{d} x^{\ell}} \psi_{k}^{n}(x), \psi_{h}^{m}(x)\right\rangle, \gamma_{k h}^{(\ell) n m}=2 \pi\left\langle\frac{\mathrm{d}^{\ell}}{\mathrm{d} x^{\ell}} \psi_{k}^{n}(x), \widehat{\psi_{h}^{m}(x)}\right\rangle
$$

where

$$
\frac{\mathrm{d}^{\ell}}{\mathrm{d} x^{\ell}} \psi_{k}^{n}(x)=(i \omega)^{\ell} \widehat{\psi}_{k}^{n}(\omega)
$$

i.e., according to (2),

$$
\frac{\mathrm{d}^{\widehat{\ell}}}{\mathrm{d} x^{\ell}} \psi_{k}^{n}(x)=-(i \omega)^{\ell} \frac{2^{-n / 2}}{2 \pi} e^{-i \omega(k+1 / 2) / 2^{n}}\left[\chi\left(\omega / 2^{n-1}\right)+\chi\left(-\omega / 2^{n-1}\right)\right] .
$$

By a direct computation it can be shown (for a sketch of the proof see [3]) 
Theorem 1. For a given $m \in \mathbb{Z} \cup\{0\}, \ell \in \mathbb{N}$, it is

$$
\begin{aligned}
& \int(i \omega)^{\ell} e^{i m \omega} d \omega=i^{\ell}(1-|\mu(m)|) \frac{\omega^{\ell+1}}{\ell+1}+ \\
& +\mu(m) e^{i m \omega} \sum_{s=1}^{\ell+1}(-1)^{[1+\mu(m)](2 \ell-s+1) / 2} \frac{\ell ! i^{\ell-s} \omega^{\ell-s+1}}{(\ell-s+1) !|m|^{s}}+\text { Cnst. }
\end{aligned}
$$

where

$$
\mu(m)=\operatorname{sign}(m)= \begin{cases}1, & m>0 \\ -1, & m<0 \\ 0, & m=0\end{cases}
$$

In particular, taking into account that

$$
e^{i k \pi}=(-1)^{k}=\left\{\begin{array}{l}
1, k= \pm 2 s \\
-1, k= \pm(2 s+1) \quad, s \in \mathbb{N}
\end{array}\right.
$$

it is

Corollary 1. For a given $m \in \mathbb{Z} \cup\{0\}, \ell \in \mathbb{N}$ and $a, b \in \mathbb{Z},(a<b)$ it is

$$
\begin{aligned}
& \int_{a \pi}^{b \pi}(i \omega)^{\ell} e^{i m \omega} d \omega=i^{\ell}(1-|\mu(m)|) \frac{\pi^{\ell+1}\left(b^{\ell+1}-a^{\ell+1}\right)}{\ell+1}+ \\
& +\mu(m) \sum_{s=1}^{\ell+1}(-1)^{[1+\mu(m)](2 \ell-s+1) / 2} \frac{\ell ! i^{\ell-s} \pi^{\ell-s+1}}{(\ell-s+1) !|m|^{s}}\left[(-1)^{m b} b^{\ell-s+1}-(-1)^{m a} a^{\ell-s+1}\right]
\end{aligned}
$$

The last formula enables us to compute the connection coefficients

Theorem 2. The any order connection coefficients (4) 2 of the Shannon wavelets (11) 2 are

$$
\begin{aligned}
& \gamma_{k h}^{(\ell) n m}=\delta^{n m}\left\{i^{\ell}(1-|\mu(h-k)|) \frac{\pi^{\ell} 2^{n \ell-1}}{\ell+1}\left(2^{\ell+1}-1\right)\left(1+(-1)^{\ell}\right)+\right. \\
& +\mu(h-k) \sum_{s=1}^{\ell+1}(-1)^{[1+\mu(h-k)](2 \ell-s+1) / 2} \frac{\ell ! i^{\ell-s} \pi^{\ell-s}}{(\ell-s+1) !|h-k|^{s}}(-1)^{-s-2(h+k)} 2^{n \ell-s-1} \times \\
& \left.\times\left\{2^{\ell+1}\left[(-1)^{4 h+s}+(-1)^{4 k+\ell}\right]-2^{s}\left[(-1)^{3 k+h+\ell}+(-1)^{3 h+k+s}\right]\right\}\right\}
\end{aligned}
$$

respectively, for $\ell \geq 1$, and $\gamma_{k h}^{(0)} \underset{k m}{n m}=\delta_{k h} \delta^{n m}$. 
Proof: It can be easily shown that (see also [3] theorem 6)

$$
\gamma_{k h}^{(\ell) n m}=0 \quad, \quad(n \neq m)
$$

When $n=m$, from equation (4) it is [3,

$$
\gamma_{k h}^{(\ell)} \underset{n n}{n n}=\frac{2^{(n-1) \ell}}{4 \pi}\left[\int_{2 \pi}^{4 \pi}(i \omega)^{\ell} e^{-i \omega(k-h) / 2} \mathrm{~d} \omega+\int_{-4 \pi}^{-2 \pi}(i \omega)^{\ell} e^{-i \omega(k-h) / 2} \mathrm{~d} \omega\right],
$$

that is, taking into account (9), equation (10) follows.

The connection coefficients fulfill some simmetries, which enable us to restrict their computation at the lowest scale, according to

Theorem 3. The connection coefficients are recorsively given by the matrix at the lowest scale level:

$$
\gamma_{k h}^{(\ell) n n}=2^{\ell(n-1)} \gamma_{k h}^{(\ell) 11}
$$

Moreover it is

$$
\gamma_{k h}^{(2 \ell+1) n n}=-\gamma_{h k}^{(2 \ell+1) n n} \quad, \quad \gamma_{k h}^{(2 \ell) n n}=\gamma_{h k}^{(2 \ell) n n} .
$$

For the coefficients of the scaling functions

$$
\lambda_{k h}^{(\ell)}=2 \pi\left\langle\frac{\mathrm{d}^{\ell}}{\mathrm{d} x^{\ell}} \varphi_{k}^{0}(x), \widehat{\varphi_{h}^{0}(x)}\right\rangle
$$

it can be also shown that

Theorem 4. The any order connection coefficients (4) 1 of the scaling functions $\varphi_{k}^{0}(x)$ are

$$
\begin{aligned}
& \lambda_{k h}^{(\ell)}=i^{\ell}(1-|\mu(h-k)|) \frac{\pi^{\ell}\left[1+(-1)^{\ell}\right]}{2(\ell+1)}+ \\
& +\mu(h-k)(-1)^{h-k} \sum_{s=1}^{\ell+1}(-1)^{[1+\mu(h-k)](2 \ell-s+1) / 2} \frac{\ell ! i^{\ell-s} \pi^{\ell-s}}{2(\ell-s+1) !|h-k|^{s}}\left[1+(-1)^{\ell-s}\right]
\end{aligned}
$$

\section{Reconstruction of a Function by Shannon Wavelets}

Let $f(x)$ be a given function such that the r.h.s. series expansion

$$
f(x)=\sum_{h=-\infty}^{\infty} \alpha_{h} \varphi(x-h)+\sum_{n=0}^{\infty} \sum_{k=-\infty}^{\infty} \beta_{k}^{n} \psi_{k}^{n}(x),
$$

converges to $f(x)$. If we limit the dilation factor $n \leq N<\infty$, for a truncated series, we have the approximation of $f(x)$, given by: 


$$
f(x) \cong \sum_{h=-S}^{S} \alpha_{h} \varphi(x-h)+\sum_{n=0}^{N} \sum_{k=-M}^{M} \beta_{k}^{n} \psi_{k}^{n}(x)
$$

with

$$
\alpha_{h} \equiv \int_{-\infty}^{\infty} f(x) \varphi(x-h) \mathrm{d} x, \quad \beta_{k}^{n} \equiv \int_{-\infty}^{\infty} f(x) \psi_{k}^{n}(x) \mathrm{d} x .
$$

By re-arranging the many terms of the series (15) with respect to the different scales, for a fixed $N$ we have

$$
f(x) \cong \sum_{h=-S}^{S} \alpha_{h} \varphi(x-h)+\sum_{n=0}^{N} f_{n}(x) \quad, \quad f_{n}(x)=\sum_{k=-M}^{M} \beta_{k}^{n} \psi_{k}^{n}(x)
$$

where $f_{n}(x)$ represent the components of the function $f(x)$ at the scale $0 \leq n \leq$ $N$, and $f(x)$ reults from a multiscale approximation.

Let us compute the approximate representation of the even function

$$
f(x)=e^{-(16 x)^{2} / 2}
$$

The bottom lenght (i.e. the main part) of the function $f(x)$ is concentrated in the interval $[-0.2,0.2]$. With a low scale $n=3$ we can have a good approximation of the function, even with a small number $k$ of translation. In fact, with $|k| \leq 5$ the absolute value of the approximation error is less than $5 \%$. Thus we can assume

$$
e^{-(16 x)^{2} / 2} \cong \alpha_{0} \varphi(x)+\sum_{n=0}^{3} \sum_{k=-5}^{5} \beta_{k}^{n} \psi_{k}^{n}(x),
$$

with $\alpha_{0}=0.155663$ and $\beta_{k}^{n}$ given by (16).

\section{Wavelet Representation of Operators}

Let $f(x)$ a function represented by (14). Any linear differential operator $L$ acting on $f$ is

$$
L f(x)=\sum_{h=-S}^{S} \alpha_{h} L \varphi_{h}^{0}(x)+\sum_{n=0}^{N} \sum_{k=-M}^{M} \beta_{k}^{n} L \psi_{k}^{n}(x)
$$

and, since

$$
L \varphi_{h}^{0}(x)=\sum_{h}\left\langle L \varphi_{k}^{0}, \varphi_{h}^{0}\right\rangle \varphi_{h}^{0}(x) \quad, \quad L \psi_{k}^{n}(x)=\sum_{m, h}\left\langle L \psi_{k}^{n}, \psi_{h}^{m}\right\rangle \psi_{h}^{m}(x)
$$

i.e.

$$
L \varphi_{h}^{0}(x)=\sum_{h} \lambda^{(\ell)}{ }_{k h}, \varphi_{h}^{0}(x) \quad, \quad L \psi_{k}^{n}(x)=\sum_{m, h} \gamma_{k h}^{(\ell) n m} \psi_{h}^{m}(x)
$$

we have, as a projection of the operator at the scale $N$ : 
a)

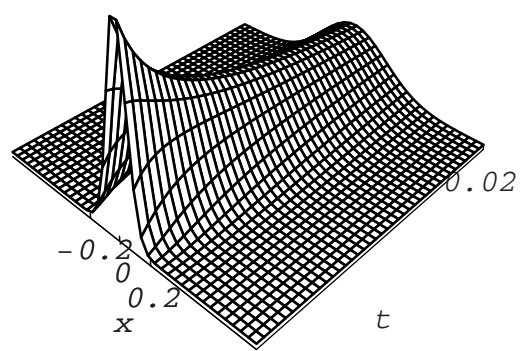

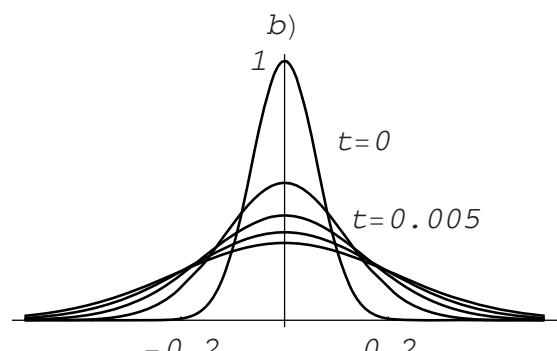

$-0.2$

0.2

Fig. 1. Surface $u(x, t)$ a) and b) time evolution of the Fourier integral solution

$$
L f(x)=\sum_{h=-S}^{S} \sum_{k} \alpha_{h} \lambda^{(\ell)}{ }_{k h}, \varphi_{k}^{0}(x)+\sum_{n=0}^{N} \sum_{k=-M}^{M} \sum_{m, h} \beta_{k}^{n} \gamma_{k h}^{(\ell) n m} \psi_{h}^{m}(x)
$$

As an example, let us consider the one-dimensional heat equation for an infinite bar, with normalized physical constants

$$
\frac{\partial u}{\partial t}=L u, \quad L \equiv \frac{\partial^{2}}{\partial x^{2}},
$$

and initial condition $(f(x)$ given by (18))

$$
u(x, 0)=f(x),-\infty \leq x \leq \infty, t=0 .
$$

The solution of the problem (21)-(22), in terms of Fourier integrals, is:

$$
u(x, t)=\frac{1}{2 \sqrt{\pi t}} \int_{-\infty}^{\infty} f(\xi) \exp \left\{-\frac{(x-\xi)^{2}}{4 t}\right\} \mathrm{d} \xi, \quad t \neq 0
$$

which, assuming (18) as initial function, can be easily computed (see Fig. 1)

$$
u(x, t)=\frac{1}{\sqrt{2^{9} t+1}} e^{-(16 x)^{2} /\left(1+2^{9} t\right)} .
$$

Thus in a short time the high peak (of the initial profile) reduces to a smooth profile. In the wavelet approach, according to the best scale-dilation approximation of the initial function (18), restricted to the interval $[-1,1]$, we can assume that at the resolution $n \leq 3,|k| \leq 5$, the solution of (21) can be expressed as

$$
u(x, t)=\alpha_{0}(t) \varphi(x)+\sum_{n=0}^{3} \sum_{k=-5}^{5} \beta_{k}^{n}(t) \psi_{k}^{n}(x)
$$


a)

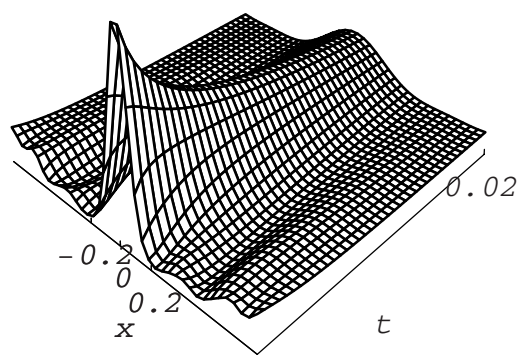

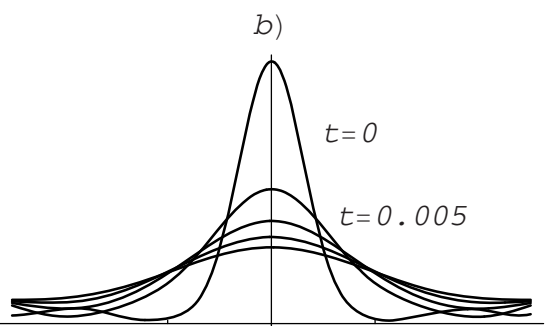

$-0.2$

0.2

Fig. 2. Surface of the approximate wavelet solution for $u(x, t)$ a) and b) its time evolution

so that

$$
\dot{\alpha}_{0}(t) \varphi(x)+\sum_{n=0}^{3} \sum_{k=-5}^{5} \dot{\beta}_{k}^{n}(t) \psi_{k}^{n}(x)=\alpha_{0}(t) L \varphi(x)+\sum_{n=0}^{3} \sum_{k=-5}^{5} \beta_{k}^{n}(t) L \psi_{k}^{n}(x)
$$

By a scalar product with scaling and wavelet basis we get $(n=0, \ldots, 3$; $k=-5, \ldots, 5)$

$$
\left\{\begin{aligned}
\frac{\mathrm{d} \alpha_{0}(t)}{\mathrm{d} t} & =\alpha_{0}(t)\left\langle\frac{\mathrm{d}^{2}}{\mathrm{~d} x^{2}} \varphi(x), \varphi(x)\right\rangle \\
\frac{\mathrm{d} \beta_{k}^{n}(t)}{\mathrm{d} t}\left\langle\psi_{k}^{n}(x), \psi_{h}^{m}(x)\right\rangle & =\sum_{m=0}^{3} \sum_{h=-3}^{3} \beta_{k}^{n}(t)\left\langle\frac{\mathrm{d}^{2}}{\mathrm{~d} x^{2}} \psi_{k}^{n}(x), \psi_{h}^{m}(x)\right\rangle,
\end{aligned}\right.
$$

i.e., taking into account (12) and (10) at the second order $(\ell=2)$, it is

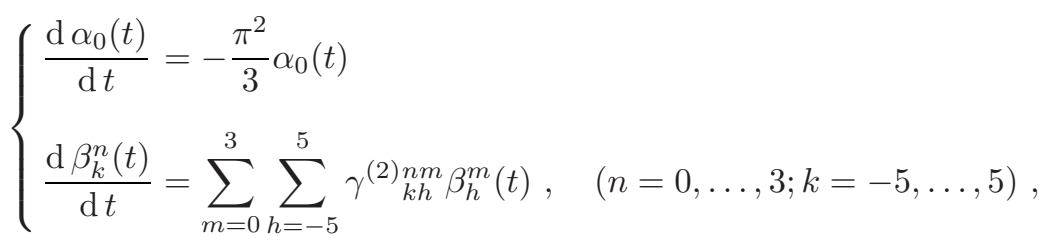

The initial conditions, coincide with the values of the wavelet coefficients of the initial profile, i.e.

$$
\alpha_{0}(0)=\left\langle f(x), \varphi_{k}^{n}(x)\right\rangle=0.155663, \beta_{k}^{n}(0)=\left\langle f(x), \psi_{k}^{n}(x)\right\rangle
$$

are given by integrals (16). Since (25) is a linear system we can easily get the solution for $\alpha$ :

$$
\alpha(t)=0.155663 e^{-\pi^{2} t / 3}
$$


as well as for the detail coefficients $\beta$, so that the solution can be (numerically) computed (see Fig. 2). Comparing the approximate wavelet solution (Fig. 2) with the exact solution (24) (Fig.1) it substantially coincides with the Fourier solution (24) even with a low number of scales. However, it should be noticed that (only) with the first approch it is possible to decompose the solution at the different scales (anologously what is done in Fourier series with mode decomposition) which is impossible to do with the Fourier integral solution (23), (24).

If we limit to the interval $[-1,1]$ we can see that the evolution of the initial profile can be split into four different waves: $f(x) \cong f_{0}(x), f(x) \cong f_{1}(x), f(x) \cong$ $f_{2}(x), f(x) \cong f_{3}(x), \quad$ (corresponding to the four scales $\left.n=0, \ldots, 3\right)$. The low frequency wave has a low amplitude but is more steady, while the higher frequency wave $(n=3)$ has higher amplitude but a quite fast decay.

\section{References}

1. C. Cattani. Harmonic Wavelet Solutions of the Schrödinger Equation. International Journal of Fluid Mechanics Research, 5, 2003, 1-10.

2. C. Cattani. Harmonic Wavelets towards Solution of Nonlinear PDE. Computers and Mathematics with Applications, 50, 8-9, 2005, 1191-1210.

3. C.Cattani, "Connection Coefficients of Shannon Wavelets", Mathematical Modelling and Analysis, vol. 11 (2),(2006), 1-16.

4. S.V. Muniandy and I.M. Moroz. Galerkin modelling of the Burgers equation using harmonic wavelets. Phys.Lett. A, 235, 1997, 352-356.

5. D.E. Newland. Harmonic wavelet analysis. Proc.R.Soc.Lond. A, 443, 1993, 203-222.

6. J.J. Rushchitsky, C. Cattani and Terletska E.V. Wavelet Analysis of a Single Pulse in a linearly Elastic Composite // International Applied Mechanics. 2005. Volume 41 (4). P. 374-380.

7. C. Toma, An Extension of the Notion of Observability at Filtering and Sampling Devices, Proceedings of the International Symposium on Signals, Circuits and Systems Iasi SCS 2001, Romania, 233-236.

8. G. Toma, Practical Test Functions Generated by Computer Algorithms, Lecture Notes Computer Science 3482 (2005), 576-585. 